\title{
Editorial
}

\section{PARP inhibitors and IR join forces to strike glioblastoma-initiating cells}

\author{
N Lugli ${ }^{1,2,4}$, I Kamileri ${ }^{2,4}$ and TD Halazonetis ${ }^{\star, 2,3}$ \\ Cell Death and Differentiation (2014) 21, 192-193; doi:10.1038/cdd.2013.172
}

Glioblastoma is the most common brain tumor in the adult, but an effective therapy still remains a major challenge. Current treatment regimens involve surgical removal of the tumor followed by radiation therapy and chemotherapy. However, overall patient survival is less than 2 years after diagnosis. ${ }^{1}$

A widely accepted hypothesis to explain the poor patient survival posits that glioblastomas contain distinct cell populations that respond differently to therapy. These populations comprise the glioblastoma-initiating cells (GICs), also referred to as glioblastoma stem cells, and the non-GICs, which constitute the majority of the tumor mass. ${ }^{2-4}$ Considerable evidence supports the notion that GICs are the tumor-initiating cells and that they have stem cell properties. In vitro, GICs form neurospheres, which can be propagated indefinitely, and when injected into mice, GICs form tumors much more efficiently than non-GICs. GICs express stem cell markers, notably PROM1 (CD133), FUT4 (SSEA-1), L1CAM and CD44, and they can also express markers of neurons, astrocytes and oligodendrocytes, suggesting that they can differentiate into all major cell types present in the adult brain. $^{2-4}$

Consistent with the hypothesis that poor patient survival is linked to the presence of a therapy-resistant cell population in glioblastoma, GICs are resistant to ionizing radiation (IR) ${ }^{5,6}$ and to the chemotherapeutic agent temozolomide, ${ }^{7}$ both of which are widely used in the clinic as first-line therapy. ${ }^{1}$ The resistance has been attributed to enhanced DNA damage checkpoint responses, enhanced repair of damaged DNA and enhanced export of toxic compounds from the cells. ${ }^{5-7}$

In an article in this issue, Venere et al. ${ }^{8}$ identified new properties of GICs that can be exploited for more efficient therapies. Specifically, they report that GICs isolated from xenografted human glioblastomas contain high levels of reactive oxygen species (ROS), at least as compared with non-GICs. In parallel, GICs contain high levels of 8-oxoguanine, single-stranded DNA and activated poly-ADP ribose polymerase 1 (PARP1), consistent with the known ability of ROS to induce DNA damage and activate PARP1. ${ }^{9,10}$

The presence of high levels of ROS in GICs was unexpected. Normal stem cells from a variety of tissues, including hematopoietic stem cells and mammary epithelial stem cells, have low levels of ROS and this is considered important for maintenance of genomic integrity. ${ }^{11,12}$ Normal neural stem cells have also been reported to have low levels of ROS. ${ }^{13-15}$ Interestingly, however, differentiation of neuronal progenitors into more differentiated cells has been associated with induction of ROS, suggesting that in the brain ROS may have a role in the development of the neuronal phenotype. ${ }^{14,15}$ In these studies, ROS production is observed primarily in the neurogenic regions of the brain, suggesting that high levels of ROS are associated with a specific stage of neuronal differentiation, yet, are absent in the stem/progenitor cells and the fully differentiated neurons. ${ }^{15}$ Somewhat contradicting these earlier studies, a more recent study proposes that ROS is present in neural stem cells, where it functions as a second messenger promoting self-renewal. ${ }^{16}$ Along the lines of the latter study, the high levels of ROS in GICs could be stimulating cell proliferation.

Irrespective of the presence and physiological significance of ROS in normal neural stem cells, the presence of ROS and activated PARP1 in GICs raises the possibility of therapeutic intervention. Indeed, the authors show that Olaparib, a wellcharacterized PARP1 and PARP2 inhibitor, ${ }^{10}$ targets GICs, inducing apoptosis and inhibiting their ability to form neurospheres. The most significant loss of viability in vitro was observed when the PARP inhibitor was combined with exposure to IR. Under these conditions, GICs were almost eliminated from the culture. Similar effects were observed in mouse xenograft models (Figure 1). PARP inhibitor monotherapy did not decrease tumor volume, IR on its own had a small effect, but the combination of Olaparib and IR led to complete elimination of the tumor, again suggesting a synergistic effect of Olaparib with IR. Importantly, in all these experiments, the authors used clinically relevant doses of Olaparib and IR. Further, as both in vitro and in vivo, the GIC population was preferentially targeted by the Olaparib/IR combination, these results imply that tumor development is driven primarily by the GICs.

The synergistic effect of Olaparib and IR is reminiscent of the synthetic lethality observed when cells with defects in the breast cancer susceptibility 1 (BRCA1) or BRCA2 genes are

\footnotetext{
${ }^{1}$ National Centre of Competence in Research Frontiers in Genetics, Geneva, Switzerland; ${ }^{2}$ Department of Molecular Biology, University of Geneva, Geneva, Switzerland and ${ }^{3}$ Department of Biochemistry, University of Geneva, Geneva, Switzerland

*Corresponding author: TD Halazonetis, Department of Molecular Biology, University of Geneva, 30, quai Ernest-Ansermet, Geneva 1205, Switzerland. Tel: +41 22379 6112; Fax: +41 22379 6868; E-mail: Thanos. Halazonetis@unige.ch

${ }^{4}$ These two authors contributed equally to this work.
} 

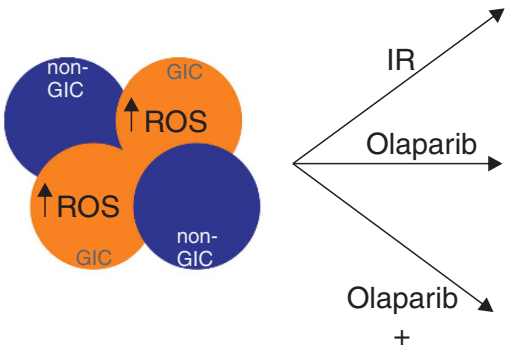

IR
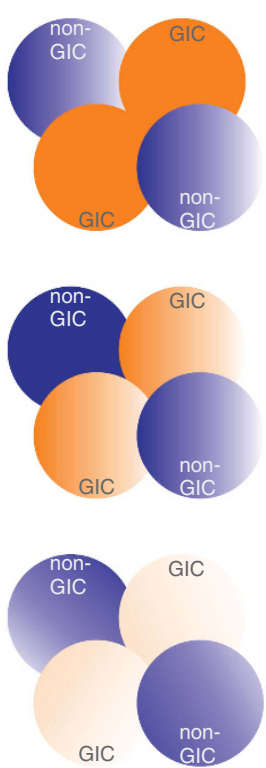

Figure 1 Synergistic effect of a PARP inhibitor (Olaparib) and IR in eliminating GICs and GIC-derived tumors. A tumor contains GICs (orange) and non-GICs (blue). A lighter color indicates elimination of the respective cell populations

treated with PARP inhibitors. ${ }^{17,18}$ BRCA1- and BRCA2deficient cells could rely on PARP1 to repair DNA damage that would otherwise be repaired by homologous recombination and, thus, are very sensitive to PARP inhibitors. ${ }^{19}$ By analogy, the presence of ROS in GICs induces genotoxic stress. IR likely potentiates the extent of DNA damage, such that irradiated GICs with compromised PARP function cannot survive.

The demonstration that cells deficient in homologous recombination are very sensitive to PARP inhibitors, generated significant excitement, as hereditary breast and ovarian cancers often lack functional BRCA1 or BRCA2 genes. ${ }^{19}$ This initial enthusiasm has been tempered by limited success in the clinic that, however, may have been because of poor selection of the patient population. Indeed, major pharmaceutical companies are now in the process of reevaluating PARP inhibitors using better defined patient populations. ${ }^{20}$ The work of Venere et al. ${ }^{8}$ opens up new opportunities for using PARP inhibitors in the clinic. Specifically, a combination of a PARP inhibitor with IR may be considered for therapy of glioblastomas. Further, it is possible that the paradigm established with glioblastomas may be relevant in other cancer types. Thus, the potential of PARP inhibitors as a cancer therapeutic still shines bright.

\section{Conflict of Interest}

The authors declare no conflict of interest.

1. Stupp R et al. Lancet Oncol 2009; 10: 459-466.

2. Singh SK, Clarke ID, Terasaki M, Bonn VE, Hawkins C, Squire J et al. Cancer Res 2003; 63: 5821-5828.

3. Galli R, Binda E, Orfanelli U, Cipelletti B, Gritti A, De Vitis S et al. Cancer Res 2004; 64: 7011-7021.

4. Yuan X, Curtin J, Xiong Y, Liu G, Waschsmann-Hogiu S, Farkas DL et al. Oncogene 2004; 23: $9392-9400$.

5. Bao S, Wu Q, McLendon RE, Hao Y, Shi Q, Hjelmeland AB et al. Nature 2006; 444: 756-760.

6. Rivera M, Sukhdeo K, Yu J. Front Oncol 2013; 3: 74

7. Liu G, Yuan X, Zeng Z, Tunici P, Ng H, Abdulkadir IR et al. Mol Cancer 2006; 5: 67.

8. Venere, Hamerlik $P$, Wu Q, Rasmussen RD, Song LA, Vasanji A et al. Cell Death Differ 2014; 21: 258-269.

9. Bertram C, Hass R. Biol Chem 2008; 389: 211-220.

10. Davar D, Beumer JH, Hamieh L, Tawbi H. Curr Med Chem 2012; 19: 3907-3921.

11. Ito K, Hirao A, Arai F, Matsuoka S, Takubo K, Hamaguchi I et al. Nature 2004; 431: 997-1002.

12. Diehn M, Cho RW, Lobo NA, Kalisky T, Dorie MJ, Kulp AN et al. Nature 2009; 458: 780-783.

13. Smith J, Ladi E, Mayer-Proschel M, Noble M. Proc Natl Acad Sci USA 2000; 97: 10032-10037.

14. Tsatmali M, Walcott EC, Crossin KL. Brain Res 2005; 1040: 137-150.

15. Tsatmali M, Walcott EC, Makarenkova H, Crossin KL. Mol Cell Neurosci 2006; 33 : 345-357.

16. Le Belle JE, Orozco NM, Paucar AA, Saxe JP, Mottahedeh J, Pyle AD et al. Cell Stem Cell 2011; 8: 59-71.

17. Bryant HE, Schultz N, Thomas HD, Parker KM, Flower D, Lopez E et al. Nature 2005; 434: 913-917.

18. Farmer H, McCabe N, Lord CJ, Tutt AN, Johnson DA, Richardson TB et al. Nature 2005; 434: 917-921.

19. Helleday T. Mol Oncol 2011; 5: 387-393.

20. Garber K. Nat Rev Drug Discov 2013; 12: 725-727. 